03

\title{
Кинетика молекул СО с учетом резонансных VE-обменов при неравновесном течении в соплах
}

\author{
(C) А.И. Мишина, Е.В. Кустова \\ Санкт-Петербургский государственный университет, \\ 199034 Санкт-Петербург, Россия \\ e-mail: e.kustova@spbu.ru, alena.i.mishina@gmail.com
}

(Поступило в Редакцию 19 июня 2017 г.)

\begin{abstract}
Исследована поуровневая кинетика молекул СО с возбужденными электронными уровнями при неравновесном течении в соплах. Учитаны три электронных терма; в системе происходят $V V$-обмены колебательной энергией внутри каждого электронного терма, $V T$-переходы колебательной энергии в поступательную и $V E$-обмены колебательной энергией между электронными термами. Численно решена система уравнений поуровневой колебательной кинетики и газодинамики, записанная в нулевом приближении метода ЭнскогаЧепмена. Особое внимание уделяется оценке влияния $V E$-обменов колебательной энергией на формирование неравновесных заселенностей колебательных уровней. Показано, что резонансные $V E$-переходы оказывают существенное влияние на процесс релаксации и в отсутствии процессов рекомбинации и диссоциации приводят к заметному изменению заселенности верхних колебательных уровней; $V E$-обмены также влияют на зависимость колебательной температуры от времени.
\end{abstract}

DOI: $10.21883 / J T F .2018 .03 .45588 .2387$

\section{Введение}

Изучение кинетики молекул СО важно по многим причинам: СО является активной средой в газодинамических лазерах; свойства этих молекул важны при решении ряда экологических задач, например для уменьшения содержания угарного газа в выхлопных трубах автомобиля; при входе космического аппарата в атмосферу Марса молекулы газа СО могут появляться в результате диссоциации $\mathrm{CO}_{2}$. Электронные уровни молекул $\mathrm{CO}$ могут влиять на плазмохимические процессы.

Изучению колебательной релаксации в двухатомных газах посвящено большое количество работ [1-8]. Однако наибольшее внимание в литературе уделяется компонентам воздуха в основном электронном состоянии: $\mathrm{N}_{2}$, $\mathrm{O}_{2}$, NO. Процессы обмена колебательной энергией в СО изучены довольно слабо; по этой теме можно указать лишь несколько работ [9-11].

В настоящей работе исследуется колебательная релаксация молекул газа СО с возбужденными электронными уровнями, подробно рассматривается процесс релаксации молекул при неравновесном течении газа в соплах. При решении задачи используется приближение поуровневой кинетики, являющееся наиболее строгой моделью неравновесной газовой динамики. Такой подход использовался ранее для исследования сверхзвукового расширения компонентов воздуха [3,12-15], однако в перечисленных работах не учитывалось возбуждение электронных степеней свободы.

Как известно, в ходе колебательной релаксации молекул газа СО при неравновесном течении газа в соплах может возникать инверсная заселенность верхних колебательных уровней. Также особенностью кинетики $\mathrm{CO}$ является наличие каналов колебательной релак- сации, связанных с резонансными переходами между электронными термами. Задача настоящей работы оценить влияние резонансных $V E$-процессов на формирование неравновесных колебательных распределений при сверхзвуковом расширении. Обычно $V E$-обмены не учитываются при моделировании, поэтому результаты настоящей работы представляют большой интерес.

\section{Постановка задачи}

Быстрое расширение газа, проходящего через сопло, моделируется с помощью квазиодномерного подхода, при этом параметры потока газа изменяются только вдоль оси сопла $x$. Газ предварительно нагревается, и за критическим сечением сопла скорость потока становится больше скорости звука, а температура и давление газа уменьшаются. Известно, что при течениях в соплах происходит смена нескольких режимов. Находящийся в форкамере газ обычно считают равновесным. В сужающейся части сопла также делаются упрощения и предполагается химическое и термическое равновесие. Как показывают расчеты, состояние газа, движущегося до критического сечения сопла, действительно оказывается близким к равновесному. При расширении потока за критическим сечением газ резко охлаждается. Энергия колебательных степеней свободы изменяется значительно медленее, чем поступательная энергия молекул, и не успевает достичь значения, соответствующего равновесному состоянию при установившейся температуре газа. Вследствие этого мы наблюдаем режим сильной колебательной неравновесности, который характеризуется инверсной заселенностью верхних колебательных состояний. Перенаселенность верхних колебательных уровней может регулироваться $V E$-переходами. 
Также заметим, что результаты численных расчетов для течений различных газов в соплах показывают, что процессы рекомбинации и диссоциации играют важную роль в формировании неравновесных колебательных распределений, и при высоких температурах в критическом сечении сопла содержание атомов достаточно высоко [14,15].

Рассмотрим течение бинарной смеси молекул газа СО и атомов С, О в сопле. Общая постановка задачи о неравновесном расширяющемся течении описана в [16], здесь же будут учитываться следующие упрощения: газ будем предполагать невязким и нетеплопроводным; течение будем считать стационарным. При этом основное внимание будет уделяться не динамике потока, а неравновесной колебательно-электронной и химической кинетике.

Запишем уравнения, характеризующие неравновесное течение молекул газа СО в приближении поуровневой кинетики:

$$
\begin{gathered}
\frac{d\left(n_{c i} v S\right)}{d x}=S\left(R_{c i}^{v i b r}+R_{c i}^{d i s s-r e c}\right), \\
\frac{d\left(n_{a} v S\right)}{d x}=-S \sum_{c, i} R_{c i}^{d i s s-r e c}, \quad a=\mathrm{C}, \mathrm{O}, \\
\rho v \frac{d v}{d x}+\frac{d p}{d x}=0, \quad \frac{d h}{d x}+v \frac{d v}{d x}=0,
\end{gathered}
$$

где $c=1-L$ и $i=0-L_{c}$ описывают соответственно электронное и колебательное состояние молекулы, $x-$ расстояние от критического сечения вдоль оси сопла, $S(x)$ - площадь сечения сопла, $n_{c i}, \rho, p, v$ и $h-$ макропараметры системы: заселенность колебательного уровня $i$ электронного состояния $c$, плотность, давление, макроскопическая скорость газа и энтальпия единицы массы соответственно. $S(x)$ является характеристикой геометрии сопла. В настоящей работе рассматривается коническое сопло с углом полураствора $21^{\circ}$; площадь сечения в расширяющейся части сопла задается уравнением: $S(x)=\pi\left(R^{*}+x \tan \theta\right)^{2}$, где $R^{*}$ - радиус критического сечения.

Система замыкается с помощью уравнения состояния и выражения для энтальпии единицы массы газа $h$, которые записываются следующим образом:

$$
p=n k_{b} T, \quad h=h_{m} Y_{m}+\sum_{a=\mathrm{C}, \mathrm{O}} h_{a} Y_{a} .
$$

Здесь $k_{b}$ - постоянная Больцмана $\left(k_{b}=\right.$ $\left.=1.3807 \cdot 10^{-23} \mathrm{~J} \cdot \mathrm{K}^{-1}\right), \quad T-$ температура газа, $n$ - числовая плотность, $h_{m}$ и $h_{a}$ - энтальпии молекул и атомов $\mathrm{C}$ и $\mathrm{O}$ соответственно, $Y_{m}$ и $Y_{a}$ - массовые доли молекул и атомов:

$$
\begin{gathered}
Y_{m}=\frac{\rho_{m}}{\rho}, \quad Y_{a}=\frac{\rho_{a}}{\rho}, \quad a=\mathrm{C}, \mathrm{O}, \\
h_{m}=\frac{7 k_{b}}{2 m_{m}} T+\frac{1}{\rho_{m}} \sum_{c, i} \varepsilon_{c i} n_{c, i}+\frac{\varepsilon_{m}}{m_{m}},
\end{gathered}
$$

$$
\begin{gathered}
h_{a}=\frac{5 k_{b}}{2 m_{a}} T+\frac{\varepsilon_{a}}{m_{a}}, \quad a=\mathrm{C}, \mathrm{O}, \\
\rho=\rho_{m}+\sum_{a=\mathrm{C}, \mathrm{O}} \rho_{a}=n_{m} m_{m}+\sum_{a=\mathrm{C}, \mathrm{O}} n_{a} m_{a},
\end{gathered}
$$

$n_{m}, n_{a}$ - числовые плотности молекул и атомов, $\rho_{m}$ и $\rho_{a}$ - плотность молекул СО и атомов С, О в смеси, $\varepsilon_{m}, \varepsilon_{a}-$ энергия образования молекул и атомов соответственно, $\varepsilon_{c i}$ - колебательно-электронная энергия молекулы СО. Подробная информация о электронных уровнях энергии дана в работе [17]. Здесь же рассматриваются три наиболее важных состояния: $X^{1} \Sigma^{+}, a^{3} \Pi$ и $A^{1} \Pi$, термы которых представлены на рис. 1 .

Для каждого электронного состояния нанесены штрихи, соответствующие отдельным колебательным уровням, а сами кривые моделируются с помощью потециала Морзе:

$$
V(r)=D\left(1-e^{-\beta\left(r-r_{c}\right)}\right)^{2}+\varepsilon_{c},
$$

где $D-$ энергия диссоциации молекул, $c-$ номер электронного терма, $\left(r-r_{c}\right)$ - изменение межъядерного расстояния относительно положения равновесия $r_{c}$, $\beta$ - параметр потенциала, $\varepsilon_{c}-$ электронная энергия. Потенциал Морзе позволяет учесть ангармоничность колебаний, при этом колебательно-электронную энергию

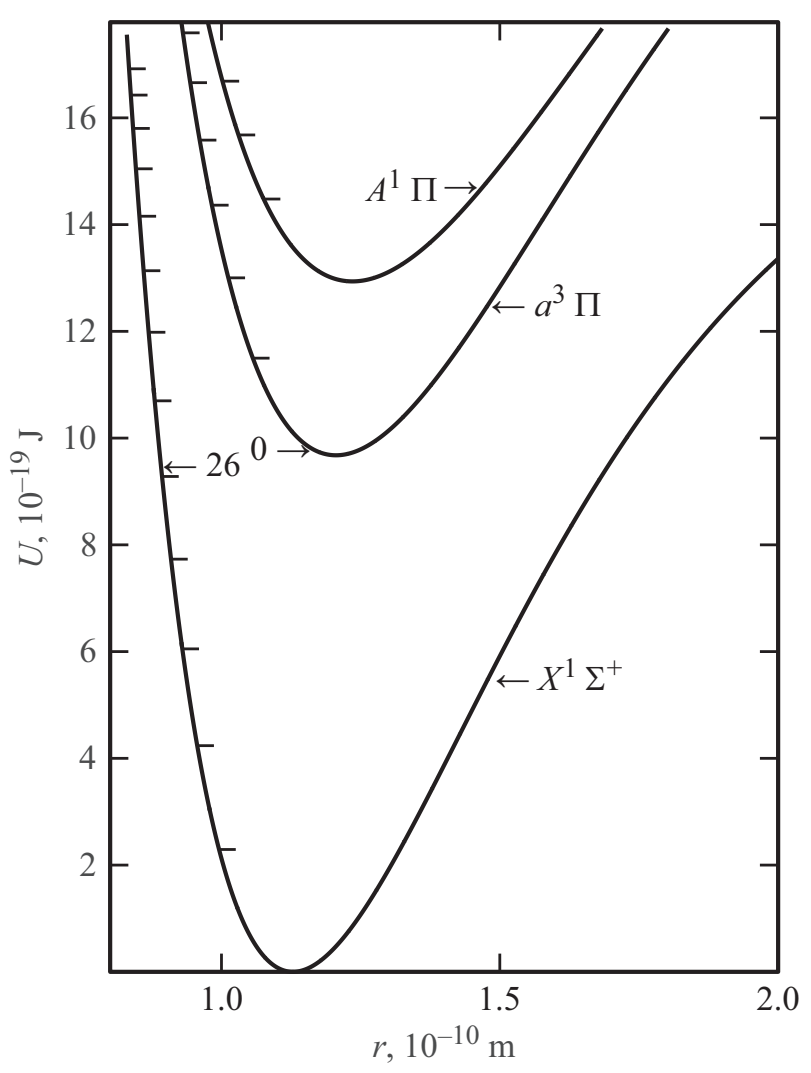

Рис. 1. Зависимость потенциальной энергии молекулы СО от межъядерного расстояния атомов. 
молекулы можно записать следующим образом:

$$
\varepsilon_{c i}=\varepsilon_{c}+\varepsilon_{i}^{c}=\varepsilon_{c}+\omega_{e}^{c}(i+1 / 2)-\omega_{e}^{c} x_{e}^{c}(i+1 / 2)^{2},
$$

где $\omega_{e}^{c}$ и $x_{e}^{c}-$ спектроскопические постоянные, $\varepsilon_{c}-$ энергия электронного уровня $c$. Число колебательных уровней каждого электронного терма определяется так, чтобы суммарная энергия не превосходила энергию диссоциации. В настоящей работе: $L=3, L_{1}=68, L_{2}=34$, $L_{3}=18$.

Уравнения (1)-(3) содержат следующие неизвестные: $n_{c i}, n_{a}, v$ и $T$, входящую в уравнения неявным образом. В этих уравнениях релаксационные члены $R_{c i}^{v i b r}$ и $R_{c i}^{d i s-r e c}$ характеризуют изменение заселенностей колебательных уровней молекул в результате различных видов энергообмена и химических реакций. Уравнения вида (1) описывают неравновесную колебательную, электронную и химическую кинетики в потоке газа.

\section{Релаксационные члены}

Релаксационные члены характеризуют изменение заселенностей колебательных уровней молекул за счет процессов, которые могут изменить колебательное состояние молекул. Такие процессы могут происходить вследствие неупругих соударений молекул. Выражение для $R_{c i}^{v i b r}$ при учете $V V$-, $V T$-, $V E$-переходов запишется следующим образом [18]:

$$
R_{c i}^{v i b r}=R_{c i}^{V V}+R_{c i}^{V T}+R_{c i}^{V E} .
$$

При $V V$-обменах происходит изменение колебательного состояния частицы при столкновениях молекул, приводящих к обмену колебательными энергиями. В настоящей работе рассматриваются одноквантовые $V V$-обмены, описывающие следующие столкновения:

$$
\mathrm{CO}(i)+\mathrm{CO}(l-1) \Leftrightarrow \mathrm{CO}(i-1)+\mathrm{CO}(l),
$$

где $i, l-1-$ номера колебательных уровней молекул до столкновения.

Изменение колебательного состояния молекул за счет столкновений, при которых одна молекула изменяет свою колебательную энергию, а у ее партнера меняется поступательная энергия, происходят при $V T$-обменах. В дальнейшем будут рассматриваться $V T$-обмены, описывающие столкновения следующего вида:

$$
\mathrm{CO}(i)+\mathrm{M} \Leftrightarrow \mathrm{CO}(i-1)+\mathrm{M},
$$

где партнер по столкновению М - молекула, которая может находиться в любом колебательно-электронном состоянии.

$V E$-обмен описывает изменение состояния молекулы вследствие столкновений, при которых происходит одновременное изменение электронного и колебательного состояний молекулы:

$$
\mathrm{CO}(c, i)+\mathrm{M} \Leftrightarrow \mathrm{CO}(d, l)+\mathrm{M},
$$

$c$ - начальное электронное состояние молекулы, $i$ начальный колебательный уровень. В дальнейшем для каждого колебательно-электронного состояния частицы будут учитываться три различных $V E$-обмена: резонансный $V E$-процесс, который характреризуется минимумом дефекта энергии $\left|\Delta \varepsilon^{v i b r-e l}\right|=\left|\varepsilon_{c i}-\varepsilon_{d k}\right|$, и два близких к резонансному. Приведем пример таких обменов: рассмотрим колебательное состояние $i=26$ основного электронного уровня $X^{1} \Sigma^{+}$. Для данного колебательноэлектронного состояния молекулы $\mathrm{CO}$ резонансным переходом является переход на нулевой колебательный уровень электронного состояния $a^{3} \Pi$, что хорошо видно на рис. 1. Значения колебательно-электронной энергии $\varepsilon_{c i}$ для $c=X^{1} \Sigma^{+}, i=26$ и $c=a^{3} \Pi, i=0$ оказываются близкими, и $\left|\Delta \varepsilon^{v i b r-e l}\right|$, характеризующая столкновения молекул, приводящих к рассматриваемому переходу, оказывается минимальной. С увеличением $\left|\Delta \varepsilon^{v i b r-e l}\right|$ вероятность $V E$-обменов резко уменьшается, что позволяет нам учитывать лишь ближайшие к резонансным обмены.

Здесь и в дальнейшем в записи релаксационных членов для $V V$ - и $V T$-обменов индекс $c$ будет опущен, так как данные процессы происходят внутри каждого электронного терма и не описывают переходы между ними. Релаксационные члены для описанных выше процессов выглядят следующим образом:

$$
\begin{gathered}
R_{c i}^{V V}=\sum_{l}\left(k_{i+1, i}^{l, l+1} n_{i+1} n_{l}+k_{i-1, i}^{l, l-1} n_{i-1} n_{l}\right) \\
-\sum_{l}\left(k_{i, i+1}^{l+1, l} n_{i} n_{l+1}+k_{i, i-1}^{l-1, l} n_{i} n_{l-1}\right), \\
R_{c i}^{V T}=n\left(k_{i+1, i} n_{i+1}+k_{i-1, i} n_{i-1}-k_{i, i+1} n_{i}-k_{i, i-1} n_{i}\right), \\
R_{c i}^{V E}=n \sum_{d}\left(k_{l-1 \rightarrow i}^{d \rightarrow c} n_{d(l-1)}+k_{l \rightarrow i}^{d \rightarrow c} n_{d l}+k_{l+1 \rightarrow i}^{d \rightarrow c} n_{d(l+1)}\right. \\
\left.\quad-k_{i \rightarrow l-1}^{c \rightarrow d} n_{c i}-k_{i \rightarrow l}^{c \rightarrow d} n_{c i}-k_{i \rightarrow l+1}^{c \rightarrow d} n_{c i}\right)
\end{gathered}
$$

где $k_{i, i-1}^{l, l+1}, k_{i, i-1}$ и $k_{i \rightarrow l}^{c \rightarrow d}-$ коэффициенты скорости соответствующих обменов.

Также в правой части уравнения (1) появляется скорость изменения заселенностей колебательных уровней молекул $-R_{c i}^{d i s-r e c}$, описывающая реакции диссоциации и рекомбинации:

$$
\mathrm{CO}(c, i) \Leftrightarrow \mathrm{C}+\mathrm{O}
$$

Выражение для релаксационных членов, возникающих вследствие процессов диссоциации и рекомбинации, имеет следующий вид:

$$
R_{c i}^{d i s s-r e c}=\sum_{d=\mathrm{CO}, \mathrm{C}, \mathrm{O}} n_{d}\left(k_{d}^{r e c, c i} n_{\mathrm{C}} n_{\mathrm{O}}-k_{d}^{c i, d i s s} n_{c i}\right) .
$$

Здесь $k_{d}^{r e c, c i}$ и $k_{d}^{c i, d i s s}-$ коэффициенты скорости данных процессов. 

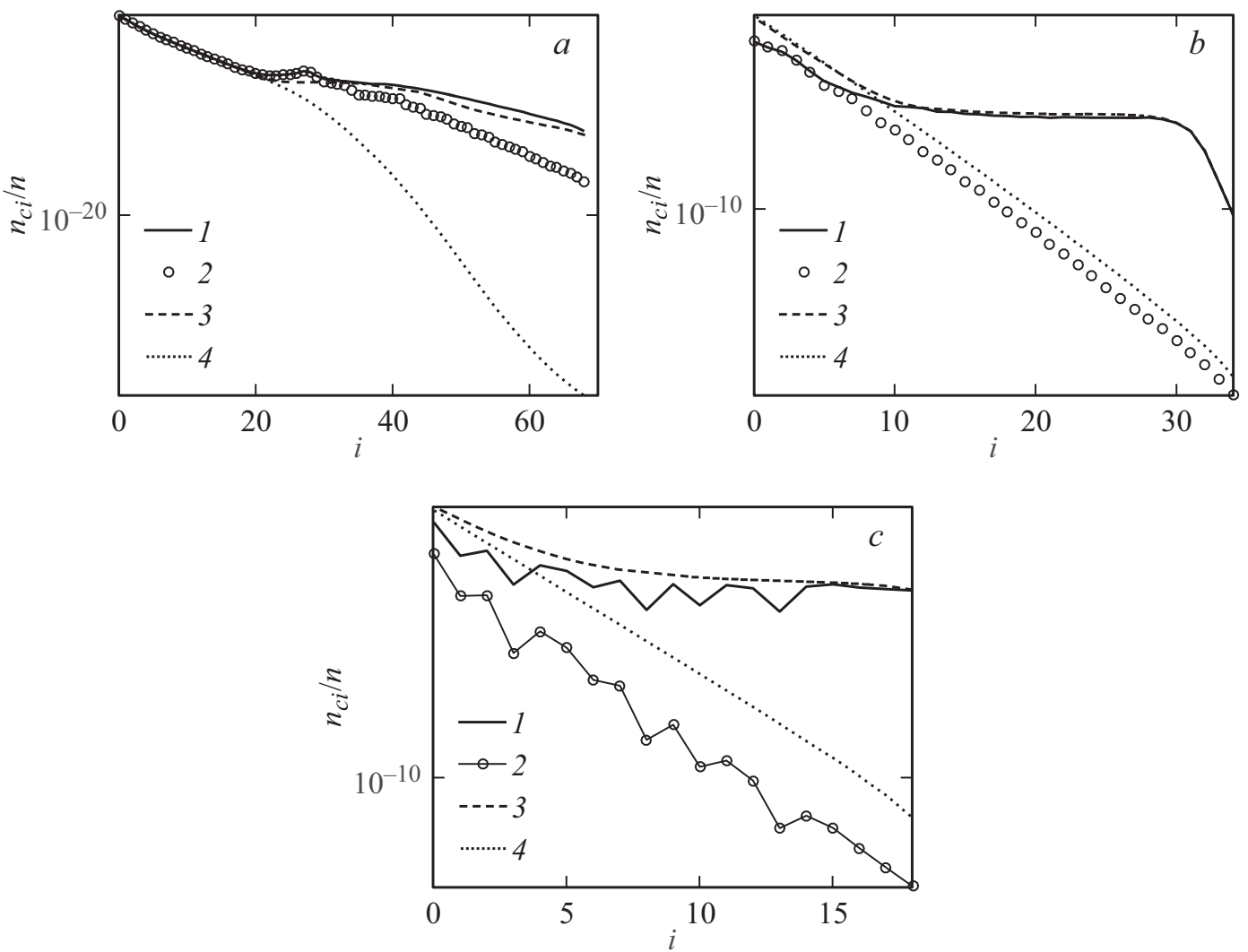

Рис. 2. Колебательные распределения для электронных термов $X^{1} \Sigma^{+}(a), a^{3} \Pi(b)$ и $A^{1} \Pi(c) .1-$ с учетом $V V$-, $V T$-, $V E$-процессов, реакций диссоциации и рекомбинации; 2 - без реакций диссоциации и рекомбинации; 3 - без учета $V E$-обменов; $4-$ с учетом только $V V$ - и $V T$-процессов.

\section{Коэффициенты скорости обменов}

Обычно в теории релаксационных процессов используются коэффициенты скорости переходов и реакций в нулевом приближении, соответствующем максвеллбольцмановскому распределению по скоростям и вращательным уровням. Эти коэффициенты определяются числом переходов в единицу времени и могут быть

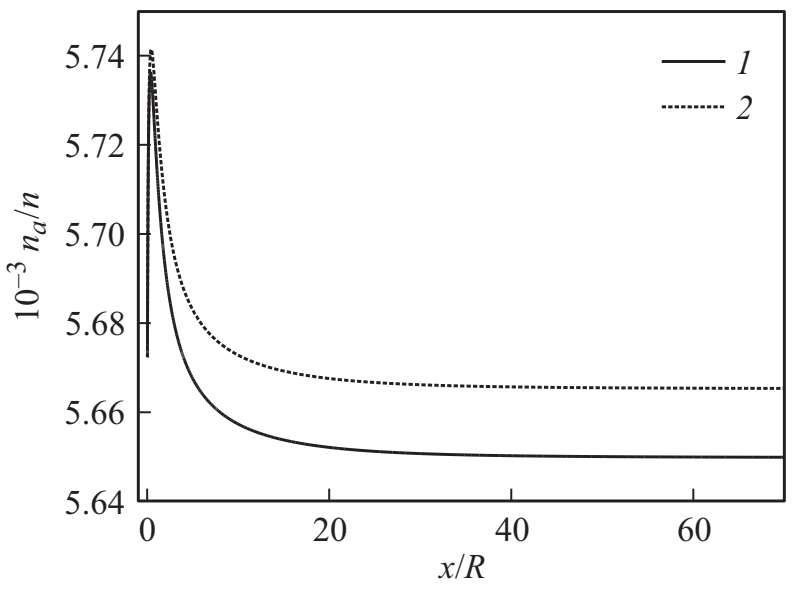

Рис. 3. Молярная доля атомов как функция $x / R .1-$ с учетом $V V$-, $V T$ - , $V E$-процессов; $2-$ с учетом $V V$-, $V T$-процессов. выражены через вероятности переходов при одном столкновении. При вычислении коэффициентов скорости различных обменов нередко используются полуэмпирические формулы, расчет по которым дает удовлетворительное согласие с экспериментом благодаря введению дополнительных параметров.

Для нахождения коэффициентов скорости $V V$ - и $V T$-обменов были использованы формулы модели Делеона [9], которая является полуэмпирической моделью, относящейся только к молекулам СО. Коэффициент скорости $V E$-обмена моделируется с помощью функции Гаусса от изменения энергии, его выражение можно найти в работе [10].

Выражения, связывающие коэффициенты скорости прямых и обратных процессов, следуют из соотношений детального баланса после их осреднения с учетом максвелл-больцмановского распределения по скоростям и вращательным энергиям.

При расчетах коэффициентов скорости рекомбинации и диссоциации чаще всего используют две модели: модель, предполагающую диссоциацию только с последнего колебательного уровня для каждого электронного состояния (лестничная модель, см. [4]), и модель ТринораМаррона [19], допускающую диссоциацию с любого колебательного уровня. В настоящей работе используется 

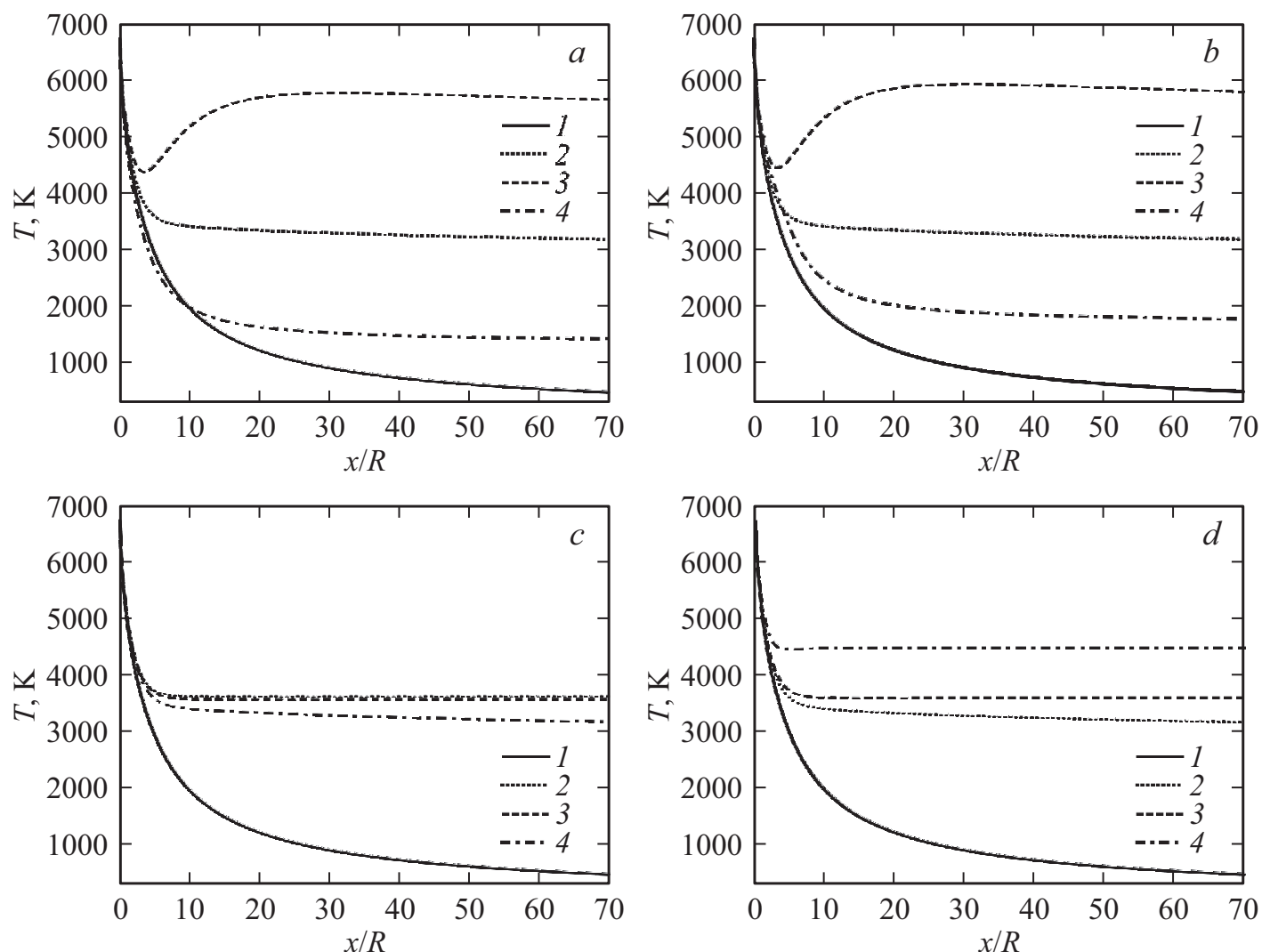

Рис. 4. Температура как функция $x / R . a-$ с учетом $V T$-, $V V$ - и резонансных $V E$-обменов; $b-$ с учетом $V T$-, $V V$-, $V E$-переходов и реакций рекомбинации и диссоциации; $c$ - с учетом $V T$ - и $V V$-процессов; $d-$ с учетом $V T$-, $V V$-переходов и реакций рекомбинации и диссоциации. $1-T, 2-T_{1}^{X^{1} \Sigma^{+}}, 3-T_{1}^{a^{3} \Pi}, 4-T_{1}^{A_{1} \Pi}$.
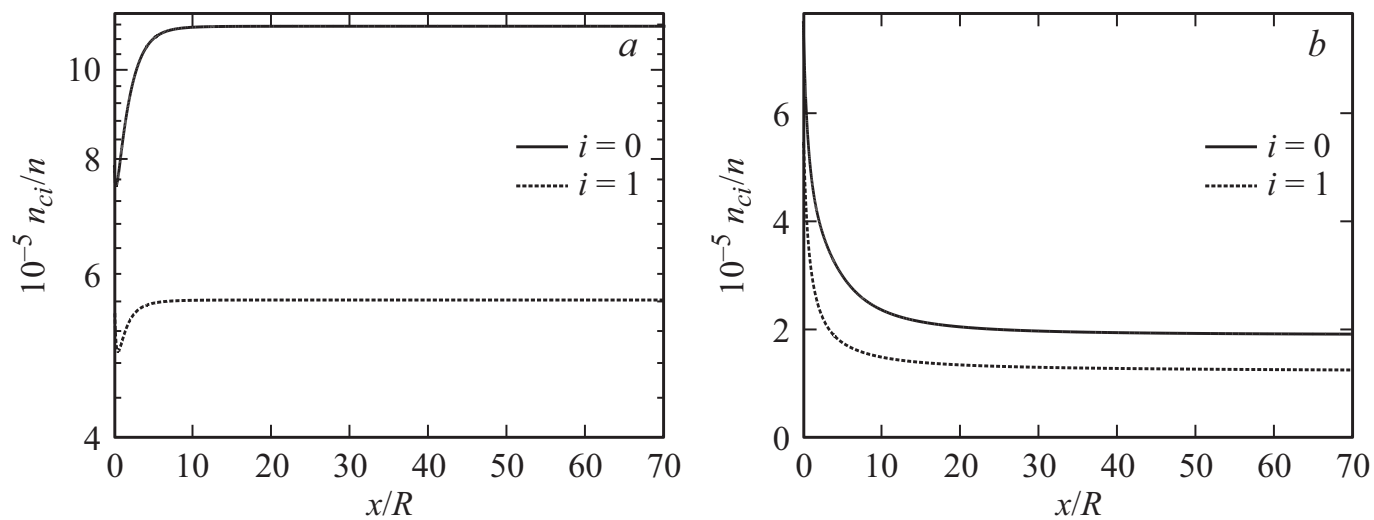

Рис. 5. Зависимость заселенностей 0 и 1 колебательных уровней от $x / R$. $a-$ с учетом $V T$-, $V V$-переходов и реакций рекомбинации и диссоциации, $b-$ с учетом $V T$-, $V V$-, $V E$-переходов и реакций рекомбинации и диссоциации.

обобщение модели Тринора-Маррона [20,21], учитывающее электронное возбуждение.

\section{Результаты}

Для численного решения системы была написана программа в среде Matlab, при интегрировании использовался встроенный метод переменного порядка с адаптивным шагом интегрирования. В этом разделе будут приведены результаты численного расчета для макропараметров системы (1)-(3). Рассмотрим коническое сопло с углом полураствора $21^{\circ}$ и радиусом критического сечения $R^{*}=1 \mathrm{~mm}$. В качестве макропараметров, соответствующих критическому сечению сопла, для системы выбраны следующие значения температуры и давления: $T^{*}=7000 \mathrm{~K}, p^{*}=10^{7} \mathrm{~Pa}$.

На рис. 2 приведена зависимость $n_{c i} / n$ от номера колебательного уровня в сечении $x / R=70$ для каж- 


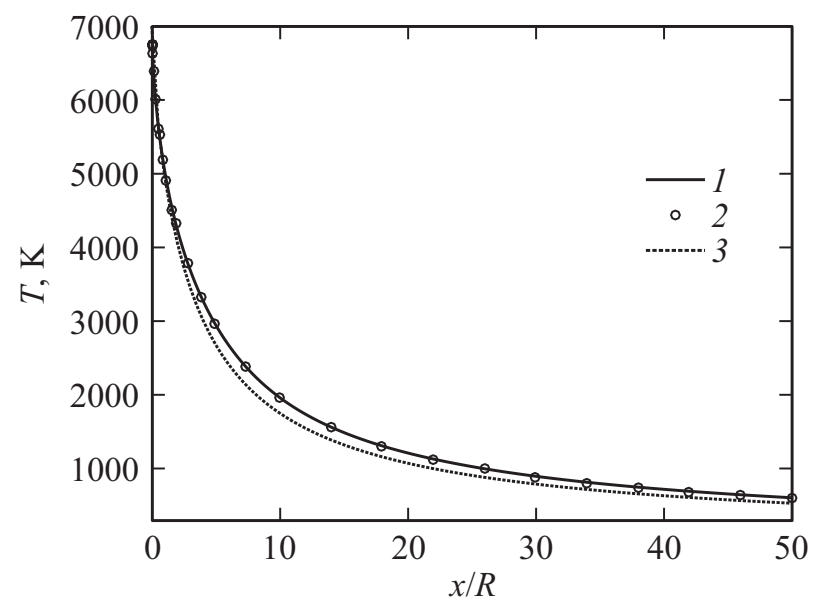

Pис. 6. Температура как функция $x / R .1-$ газ СО (учитываются $V V$-, $V T$-, $V E$-процессы и реакции диссоциации и рекомбинации), 2 - газ СО (без учета процессов диссоциации и рекомбинации), 3 - газ $\mathrm{N}_{2}$.

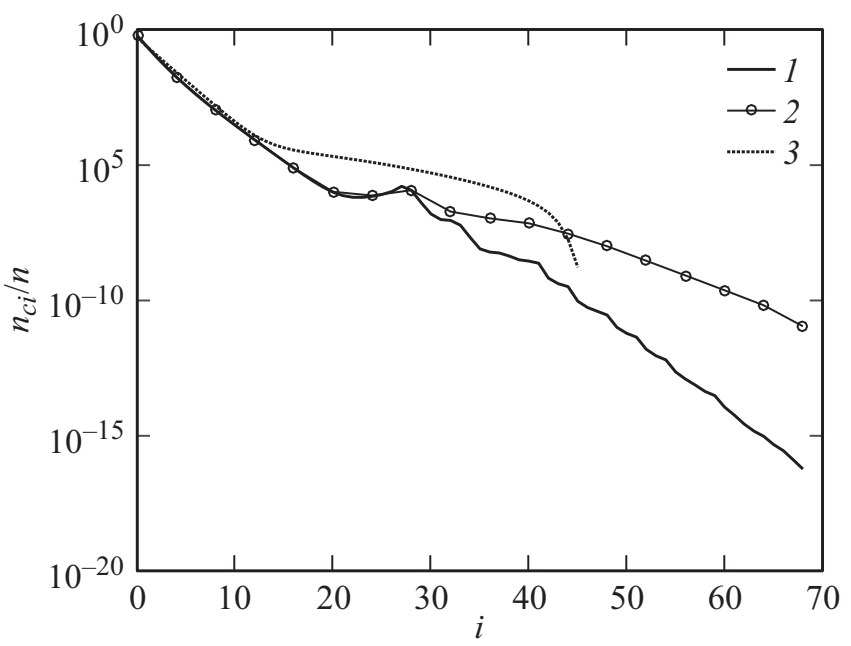

Рис. 7. Колебательные распределения. 1 - газ СО (учитываются $V V$-, $V T$-, $V E$-процессы и реакции диссоциации и рекомбинации), 2 - газ СО (без учета процессов диссоциации и рекомбинации), 3 - газ $\mathrm{N}_{2}$.

дого электронного состояния. Расчет проводился при включении различных процессов. Можно заметить, что вклад $V E$-обменов в процесс формирования неравновесных распределений значителен, и при учете переходов молекул между электронными термами инверсность заселенностей верхних колебательных уровней каждого электронного состояния становится более выраженной.

Как говорилось ранее, при рассмотрении течения газа в сопле важно учитывать процессы диссоциации и рекомбинации. Перенаселенность верхних колебательных уровней может регулироваться с помощью данных процессов. Рассмотрим рис. 3, показывающий изменение количества атомов, которое происходит при учете реакций диссоциации и рекомбинации, в зависимости от $x / R$. При высоких температурах, соответствующих участку сразу за критическим сечением сопла, в результате процесса диссоциации значение числовой плотности $n_{a}$ возрастает. Но резкое охлаждение газа приводит к активации реакций рекомбинации, что характеризуется промежутком, на котором график $n_{a}(x / R)$ начинает убывать. Заметим, что при учете $V E$-обменов вклад процессов рекомбинации становится более существенным, что также прослеживается на рис. 2.

В случаях колебательной неравновесности представляет интерес поведение температуры первого колебательного уровня $T_{1}^{c}=\varepsilon_{c 1} /\left[k_{b} \ln \left(n_{c 0} / n_{c 1}\right)\right]$. Рассмотрим рис. 4, $a$, на котором отображены результаты расчета, учитывающие $V T$ - , $V V$ - и резонансные $V E$-обмены колебательной энергии, и рис. $4, b$, где учитываются $V T$ ,$V V$-, $V E$-переходы и реакции рекомбинации и диссоциации. На рис. 4, $c$ и $d$ аналогичные графики, но без учета $V E$-переходов между термами. Заметим, что зависимость $T_{1}^{c}(x / R)$ может проявлять немонотонный характер, что объясняется различием в поведении колебательных заселенностей $n_{c 0}, n_{c 1}$ без учета $V E$-процессов и при введении в рассмотрение резонансных $V E$-обменов. Покажем это различие графически для электронного состояния $a^{3} \Pi$.

На рис. 5, $a$ представлены зависимости $n_{c 0}(x / R)$ и $n_{c 1}(x / R)$, полученные при расчете, не учитывающем резонансные $V E$-обмены; на рис. 5, $b$ учет $V E$-процессов был произведен. Заметим, что на рис. $5, b$ графики $n_{c 0}(x / R)$ и $n_{c 1}(x / R)$ на промежутке $x / R=0$-20 убыва-
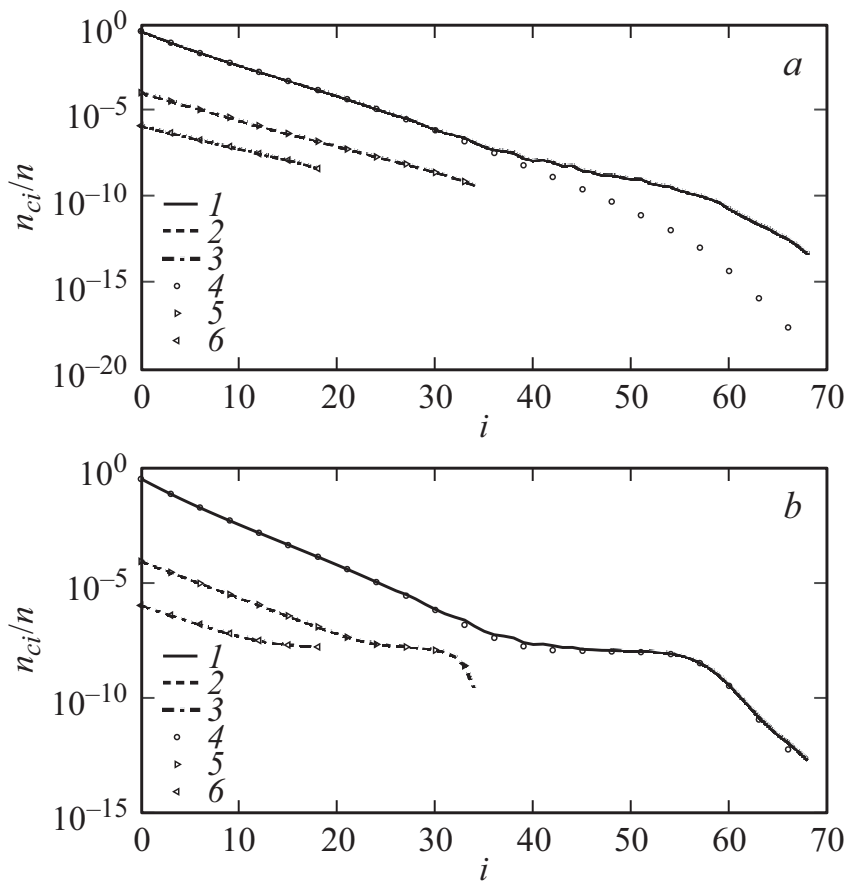

Рис. 8. Колебательные распределения, полученные без учета реакций диссоциации, рекомбинации $(a)$ и с ними $(b) .1,2$ и 3 - при учете только $V V$ - и $V T$-процессов для электронных уровней $X^{1} \Sigma^{+}, a^{3} \Pi$ и $A^{1} \Pi$ соответственно; 4, 5, $6-$ с учетом $V V$-, $V T$ - и резонансных $V E$-обменов для электронных уровней $X^{1} \Sigma^{+}, a^{3} \Pi$ и $A^{1} \Pi$ соответственно. 

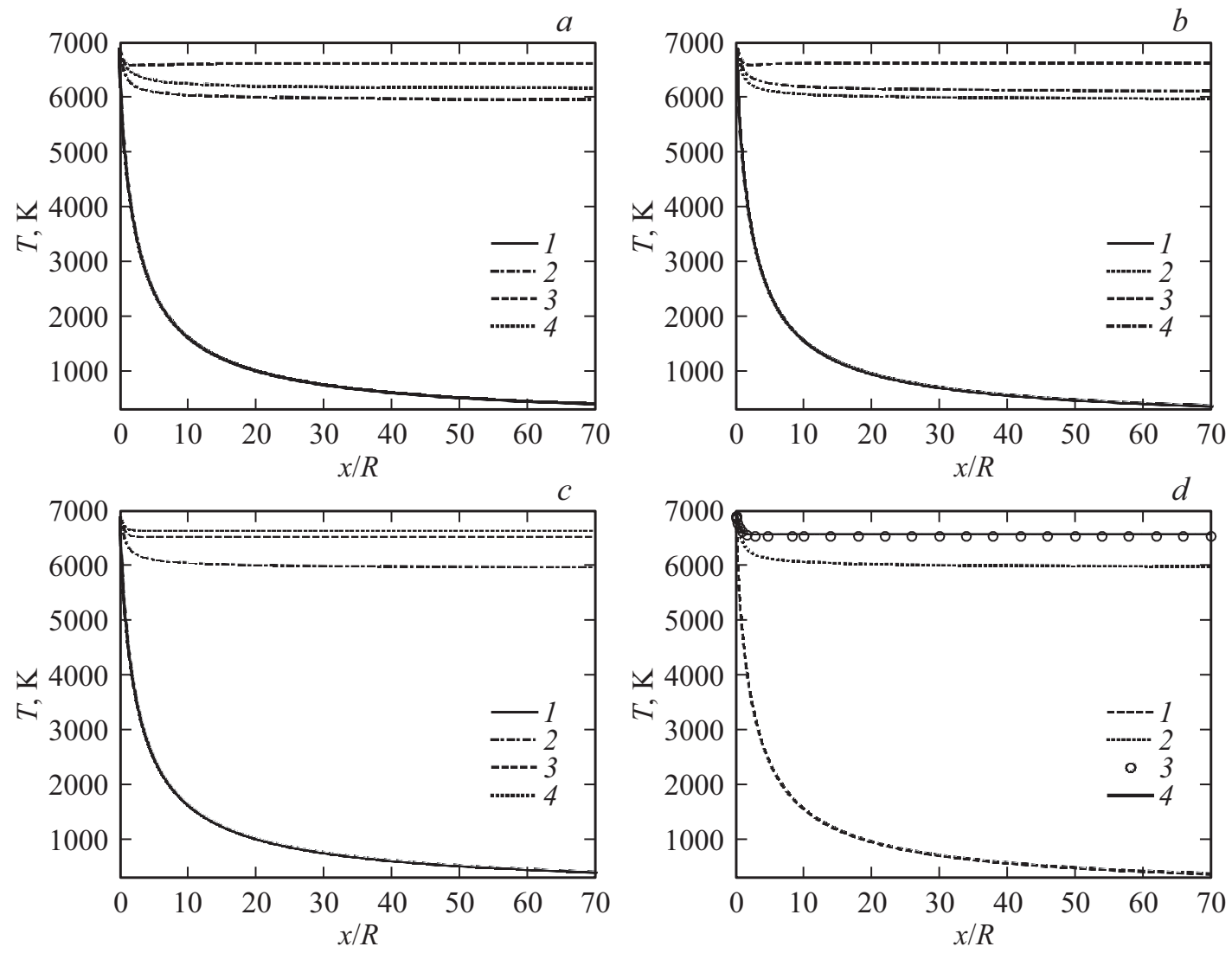

Рис. 9. Температура как функция $x / R . a-$ с учетом $V T$-, $V V$ - и резонансных $V E$-обменов, $b-\mathrm{c}$ учетом $V T$-, $V V$-, $V E$-переходов и реакций рекомбинации и диссоциации, $c-$ с учетом $V T$-и $V V$-процессов, $d-$ с учетом $V T$-, $V V$-переходов и реакций рекомбинации и диссоциации. $1-T, 2-T_{1}^{X^{1} \Sigma^{+}}, 3-T_{1}^{a^{3} \Pi}, 4-T_{1}^{A^{1} \Pi}$.

ют, но отношение этих величин ведет себя немонотонным образом, а именно данное отношение регулирует вид зависимости $T_{1}^{c}(x / R)$.

Решению задачи о неравновесном течении газов в соплах посвещено большое количество литературы. Заметим, однако, что основная часть работ содержит описание кинетики азота. Структура молекул $\mathrm{N}_{2}$ и угарного газа во многом схожа. В частности, молекула СО имеет тройную связь, как и молекула азота $\mathrm{N}_{2}$. Так как эти молекулы сходны по строению, то и свойства их также схожи.

Сравним данные, полученные при решении системы (1)-(3), с результатами вычислений, приведенными в работе [14]. В настоящей работе рассматривалось неравновесное течение смеси $\mathrm{N}_{2} / \mathrm{N}$ в коническом сопле с углом полураствора $21^{\circ}$. В критическом сечении сопла были выбраны следующие условия для макропараметров: $T^{*}=7000 \mathrm{~K}, p^{*}=10^{7} \mathrm{~Pa}$. При решении задачи использовалось приближение поуровневой кинетики.

Рис. 6 демонстрирует, что различие в поведении температуры газа, рассчитаной вдоль оси сопла, для газов $\mathrm{CO}$ и $\mathrm{N}_{2}$ крайне незначительно. Также можно заметить схожесть в зависимости колебательных распределений от номера $i$. На рис. 7 мы видим, что в сечении $x / R=50$ для нижних колебательных уровней молекул $\mathrm{CO}$ и $\mathrm{N}_{2}$ были получены близкие значения. Графики приведены для молекул $\mathrm{CO}$, находящихся в основном электронном состоянии $X^{1} \Sigma^{+}$.

Рассмотрим другие условия в критическом сечении сопла: $T^{*}=7000 \mathrm{~K}, p^{*}=10^{5} \mathrm{~Pa}$. Для заданных величин можно построить аналогичные графики, приведем некоторые из них.

Рис. 8, $а$ получен без учета диссоциации и рекомбинации; на рис. $8, b$ данные процессы учтены. Заметим, что влияние $V E$-процессов уже менее заметно, но различие колебательных заселенностей основного электронного состояния $X^{1} \Sigma^{+}$остается ощутимым. Менее выражен вклад $V E$-процессов в зависимость $T_{1}^{c}(x / R)$. На рис. $9, a$ отображены результаты расчета, учитывающие $V T$-, $V V$ - и резонансные $V E$-обмены колебательной энергии; $9, b-V T-, V V$-, $V E$-переходы и реакции рекомбинации и диссоциации; на рис. $9, c$ и $d$ представлены аналогичные графики, но без учета $V E$-переходов между термами. Обратим внимание, что при учете $V E$-процессов мы получаем различие в температурах $T_{1}^{c}$ для электронных состояний $a^{3} \Pi$ и $A_{1} \Pi$, в то время как без учета резонансных $V E$-обменов эти колебательные температуры близки. Немонотонность поведения $T_{1}^{c}(x / R)$ сохраняется, хотя становится менее заметной. 


\section{Заключение}

В настоящей работе исследовалось влияние $V E$-обменов колебательной энергией между электронными термами на процесс колебательной релаксации молекул газа СО. Подробно было рассмотрено неравновесное течение газа в соплах. В рамках данной задачи было проведено исследование влияния резонансных $V E$-процессов, реакций диссоциации и рекомбинации на формирование неравновесных распределений в сопле и поведение колебательной температуры $T_{1}^{c}$. Отметим, что без учета процессов диссоциации и рекомбинации влияние $V E$-обменов на колебательные заселенности оказалось велико; при учете рекомбинации и диссоциации вклад резонансных $V E$-переходов становится менее значимым. Также учет переходов между электронными термами приводит к интересной особенности: немонотонному поведению колебательных температур.

Работа выполнена при поддержке СПбГУ (проект 6.37.206.2016) и РФФИ (проект 15-08-03371).

\section{Список литературы}

[1] Гордиеи Б.Ф., Осипов А.И., Шелепин Л.А. Кинетические процессы в газах и молекулярные лазеры. М.: Наука, 1980. $512 \mathrm{c}$.

[2] Нагнибеда Е.А., Кустова Е.В. Кинетическая теория процессов переноса и релаксации в потоках неравновесных реагирующих газов. СПб.: Изд-во СПб. ун-та, 2003. 272 с.

[3] Shizgal B., Lordet F. // J. Chem. Phys. 1996. Vol. 104. N 10. P. 3579-3597.

[4] Capitelli M., Armenise I., Gorse C. // J. Thermophys. Heat Transfer. 1997. Vol. 11. N 4. P. 570-578.

[5] Adamovich I.V., Macheret S.O., Rich J.W., Treanor C.E. // J. Thermophys. Heat Transfer. 1998. Vol. 12. N 1. P. 57-65.

[6] Кунова О.В., Нагнибеда Е.A. // Вестник СПб. ун-та. Сер. 1. Математика, механика, астрономия. 2014. Т. 1 (59). Вып. 1. C. 124-133.

[7] Kunova O., Nagnibeda E. // Chem. Phys. 2014. Vol. 441. P. 66-76.

[8] Мишин Л.Д., Кустова Е.В. // Вестник СПб. ун-та. Сер. 1. Математика, механика, астрономия. 2016. Т. 3 (61). Вып. 2. C. 300-308.

[9] Deleon R., Rich J.W. // Chem. Phys. 1986. Vol. 107. N 2. P. 283-292.

[10] Chiroux de Gavelle de Roany A., Flament C., Rich J.W. et al. // AIAA J. 1983. Vol. 31. N 1. P. 119-128.

[11] Aliat A., Chikhaoui A., Kustova E.V. // Phys. Rev. E. 2003. Vol. 68. P. 056306.

[12] Colonna G., Capitelli M., Tuttafesta M., Giordano D. // JTHT. 1999. Vol. 13. N 3. P. 372-375.

[13] Chikhaoui A., Nagnibeda E.A., Kustova E.V., Alexandrova T.Yu. Chem. Phys. 2001. Vol. 263. P. 111-126.

[14] Kustova E.V., Nagnibeda E.A., Alexandrova T.Yu., Chikhaoui A. Chem. Phys. 2002. Vol. 276. N 2. P. 139-154.

[15] Kustova E.V., Nagnibeda E.A., Alexandrova T.Yu., Chikhaoui A. Chem. Phys. Lett. 2003. Vol. 377. N 5-6. P. $663-671$.
[16] Пирумов У.Г., Росляков Г.С. Течения газа в соплах. М.: Изд-во Моск. гос. ун-та, 1978. 288 с.

[17] Paul H. Krupenie. The Band Spectrum of Carbon Monoxide. Institute for Basic Standards National Bureau of Standards Washington, D.C., 1966. 95 p.

[18] Mishina A.I., Kustova E.V. // Vestnik St.-Peterb. Univ. 2017. Vol. 50. N 2. P. 88-197.

[19] Marrone P.V., Treanor C.E. // Phys. Fluid. 1963. Vol. 6. N 9. P. 1215-1221.

[20] Aliat A., Kustova E.V., Chikhaoui A. // Chem. Phys. Lett. 2004. Vol. 390. N 4-6. P. 370-375.

[21] Aliat A., Kustova E.V., Chikhaoui A. // Chem. Phys. 2005. Vol. 314. P. 37-47. 